\title{
Effects of aerobic training, resistance training, or both on circulating irisin and myostatin in untrained women
}

\author{
Ramin Shabani ${ }^{1}$ and Fatemeh Izaddoust ${ }^{2, *}$ \\ ${ }^{1}$ Faculty of Humanities, Islamic Azad University, Rasht Branch, Rasht, Iran; and ${ }^{2} Y o u n g$ Researchers and Elite Club, \\ Islamic Azad University, Rasht Branch, Rasht, Iran
}

Copyright: (c) 2018 R. Shabani and F. Izaddoust. This is an open access article licensed under the Creative Commons Attribution License (http://creativecommons.org/licenses/by/4.0/).

\begin{abstract}
Background: Scientific literature suggests that different types of training can have different effects on secretion of irisin and myostatin. Objectives: The purpose of this study was to determine the effect of resistance exercise training (RET), aerobic exercise training (AET) and concurrent (aerobic + resistance) exercise training (CET) on serum irisin and myostatin in untrained women. Methods: Thirty-five non-obese, untrained women were randomly assigned to control ( $n=6$, age $25.50 \pm 4.80$ years), RET ( $n=10$, age $24.60 \pm 2.45$ years), AET ( $n=9$, age $24.66 \pm 2.29$ years) or CET ( $n=10$, age $26.60 \pm 4.00$ years) groups. Subjects in training groups trained for 8 weeks, three times per week. The levels of serum irisin, myostatin were measured before and after the training period. Results: It was found that the CET group had significantly reduced serum irisin $(p=.028)$ and myostatin $(p=.005)$ concentrations. The myostatin concentration was also significantly decreased in the RET group $(p=.008)$. Conclusion: CET, AET or RET were not effective for improving (increase) irisin circulating level. In addition, RET and CET were more effective than AET in reducing circulating myostatin levels.
\end{abstract}

Keywords: strength, exercise, endurance, young

\section{Introduction}

Skeletal muscle has recently been recognized as an endocrine organ that produces and releases various cytokines termed myokines (Karsenty \& Olson, 2016) that are involved in the regulation of several physiological and metabolic pathways. Discovery of myokines has emphasized the role of muscle as an important source of exercise-induced hormones to communicate information and interact with other tissues, including fat, the liver, and the pancreas, to alter metabolism (Huh et al., 2012). Irisin is a novel glycosylated polypeptide hormone derived from its precursor fibronectin type III domain containing protein 5 (FNDC5) (Boström et al., 2012). There is controversial evidence on the physiology of irisin, but it has been suggested that under some conditions, such as exercise and elevated oxidative stress an increased expression of FNDC5 occurs and therefore of the circulating irisin levels (Kurdiova

\footnotetext{
* Address for correspondence: Fatemeh Izaddoust, Department of Exercise Physiology, Faculty of Humanities, Islamic Azad University, Rasht Branch, Taleshan Bridge St., 4147654919, Rasht, Iran. E-mail: fa.izaddoust@gmail.com
}

et al., 2014; Sanchis-Gomar \& Perez-Quilis, 2014). Several physiological functions have been identified for irisin. For example, in white adipose tissue irisin uncouples the mitochondrial respiratory chain and induces "browning". Browning of white adipose tissue increases basic energy expenditure (Spiegelman, 2013) and result in an improvement of adiposity and glucose homeostasis (Boström et al., 2012).

Furthermore, skeletal muscle secretes other hormones such myostatin, a member of the transforming growth factor $\beta$ superfamily, which is a critical factor in regulating irisin. It has been shown, irisin treatment significantly decreased myostatin gene expression in human myocytes (Huh, Dincer, Mesfum, \& Mantzoros, 2014). In addition, myostatin negatively affect muscle growth and development (McPherron, Lawler, \& Lee, 1997). It has been shown that myostatin inhibits muscle cell proliferation, protein synthesis, and differentiation (Rodgers \& Garikipati, 2008). In addition, myostatin associated with metabolism. The animal model has been shown that myostatin deficiency in mice was concurred with an increased enzymatic activity of enolase, a key component of glycolysis. While, the hyper muscular state of myostatin knockout mice 
increases oxygen consumption and the energy cost of running (Mouisel et al., 2014).

While a large body of literature exists regarding the effects of chronic exercise training on irisin in adults, the results of many studies have been far from conclusive, and no consensus has been reached. Some studies have shown the potential effects of exercise training on circulating irisin (Boström et al., 2012; Ercan et al., 2017; Ghanbari-Niaki et al., 2018; Kim, So, Choi, Kang, \& Song, 2015; Moienneia \& Hosseini, 2016; Zhao, Su, Qu, \& Dong, 2017) and myostatin (Hinkley, Konopka, Suer, \& Harber, 2017; Hittel et al., 2010; Roth et al., 2003; Saremi et al., 2010; Walker, Kambadur, Sharma, \& Smith, 2004). While others have shown that irisin (Ellefsen et al., 2014; Hecksteden et al., 2013; Moienneia \& Hosseini, 2016; Pekkala et al., 2013) and myostatin (Besse-Patin et al., 2014; Binns, Gray, Henson, \& Fort, 2017; Hofmann et al., 2016; Ziaaldini et al., 2015) may not change after long-term exercise training.

On the other hand, the authors hypothesized that different types of exercise training might result in different result on circulating levels of these myokines. Unfortunately, previous studies have not included a simultaneous comparison of aerobic exercise training (AET), resistance exercise training (RET), and concurrent $(\mathrm{AET}+\mathrm{RET})$ exercise training $(\mathrm{CET})$ on circulating irisin and myostatin. Accordingly, the aim of the present study is to compare the effects of several types of training on circulating irisin and myostatin and their correlation with each other in untrained women.

\section{Methods}

\section{Participants}

Thirty-seven non-obese healthy women who participated in the study were randomly divided into four study groups: (1) the RET group $(n=10)$, (2) the AET group $(n=9)$, (3) the CET group $(n=10)$, and (4) the control group $(n=8)$. One participant in the AET group and two in the control group withdrew from the study. Sample size for the present study was determined based on previous studies, which were conducted on healthy adults (Pekkala et al., 2013; Roth et al., 2003). The participants were recreationally physically active but without systematic strength or endurance training for at least one year prior to participation. Exclusion criteria included history of smoking, metabolic disorder, or orthopedic problems that could limit participation in the exercise protocols. The participants were instructed to maintain consistent dietary and lifestyle habits over the course of the study. Having informed the participants about the study and its procedures, written consent was obtained from all of them. The study protocol conforms to the ethical guidelines of the 1975 Declaration of Helsinki as reflected in a priori approval by the institution's human research committee. Ethical approval was obtained from the Islamic Azad University-Rasht Branch Ethics Committee (IR. IAU.RASHT.REC.1395.3) and the study was registered on the Iranian Registry of Clinical Trials website (http://www.irct.ir/, IRCT2016123125449N2).

\section{Experimental design}

Blood samples were taken from the participants before primary physical assessment, with 12 hours of fasting. All blood measurements were conducted between the 5 th and 12th day of follicular phase. Once the blood sample was collected, Subjects were familiarized with the testing protocols and exercises. Then, the participants completed three sessions with a 48 hour interval between sessions for assessment of anthropometric data, determination of one repetition maximum (1RM) loads for leg press, and maximum oxygen consumption $\left(\mathrm{VO}_{2 \max }\right)$ for Bruce treadmill test. RET, AET or CET began the week after baseline assessments, and 8 weeks later, the same measurements were repeated.

\section{Training protocol}

The exercise groups exercised for 8 weeks and participants exercised three times per week for $65 \mathrm{~min} / \mathrm{ses}$ sion with 48 hours of minimal interval between them. Each exercise session including a 10 min warm up, 50 min RET, AET, or CET and 5 min cool down.

The RET program was based on the recommendations of the American College of Sports Medicine (ACSM) for RET in healthy adults including 3-4 sets, 8-12 repetitions, 30-60 second rest interval between 2 sets, and a 2-3 minute rest between exercises (Ratamess et al., 2009). Each resistance training session consisted of nine exercises that were performed as follows: leg press, chest press, latissimus dorsi pull down, leg extension, leg curl, cable crossover, biceps curl, triceps extension, and abdominal crunches. In session one, the workload for each exercise was equal to $55 \%$ of each individual's $1 \mathrm{RM}$ consisting of $8-10$ repetitions per set. In sessions two and three, the workload was increased to $60 \%$ and $65 \%$ of $1 \mathrm{RM}$, respectively. Over 2-4 weeks, the workload was increased to $65-70 \% 1 \mathrm{RM}$, and in the second month, repetitions and load were changed to $10-12 \%$ and $70-75 \% 1 \mathrm{RM}$, respectively. All resistance exercises were performed on mobarez weight equipment (Mobarez, Tehran, Iran).

For the AET group, the researchers used the recommendations (intensity and duration in week) proposed by the American College of Sports Medicine (Garber et al., 2011). AET included 4-8 rhythmic aerobic 
step training (which includes A-step, K-step, Across The Top, Around The World, Basic Left, Basic Right, Charleston, Corner to Corner, Flamingo, Indecision, L-step, Repeater, X-step, V-step, Tap Up, Split Basic and Reverse Turn) and running at $60-75 \%$ age-predicted maximum heart rate (220 - age). In the first week of the experiment, the participants were trained at $55-65 \%$ of maximum heart rate. The training intensity progressively increased to $70 \%$ of maximum heart rate in weeks 2-4. Exercise intensity increased every two weeks to reach $75 \%$ of maximum heart rate in the second month.

The CET consisted of 25 min resistance training that was followed by $25 \mathrm{~min}$ aerobic training. Both types of training were similar to other groups; however, RET contained five exercises in each session (which included leg press, cable crossover, biceps curl, triceps extension and abdominal crunches or chest press, latissimus dorsi pull down, leg extension, leg curl, and abdominal crunches).

\section{Body composition}

Body weight was determined with an accuracy of $100 \mathrm{~g}$ on a SECA scale (SECA, Hamburg, Germany) and height was determined with an accuracy of $0.100 \mathrm{~cm}$ with a SECA stadiometer; from these values, body mass index was calculated. Waist circumference was measured using a non-stretchable tape measure, with measurements made halfway between the lower border of the ribs, and the iliac crest in a horizontal plane. Hips were measured as the widest circumference over the buttocks and below the iliac crest.

\section{Leg press strength}

Leg press strength was measured using a $1 \mathrm{RM}$ test on day one. At the start of the session, the participants warmed up series of 6 to 10 repetitions using approximately $50 \%$ of the maximum weight. After two minute of rest, the participants $1 \mathrm{RM}$ were determined by performing the most of repetitions until the offered resistance was impossible. Between 7 and 10 repetition was accepted (Nascimento et al., 2007) and each subject performed a maximum of 5 attempts with intervals of 3-5 minutes between them to reach considered repetition (Bastos et al., 2013). The Brzycki equation was used for the estimation of the 1RM (Nascimento et al., 2007).

\section{$\mathrm{VO}_{2 \max }$}

Subjects completed a standardized treadmill test (Bruce, Kusumi, \& Hosmer, 1973) on a motorized treadmill (Sportek Industrial, Taiwan) (Green, Crews, Bosak, \& Peveler, 2003). The Bruce test is a continuous, incremental protocol where treadmill speed and grade are increased at every stage $(3 \mathrm{~min})$, until the participant reaches exhaustion. Criteria for achievement of exhaustion were: (a) heart rate at test termination $\geq 85 \%$ of age-predicted maximum heart rate, and (b) rate of perceived exertion (RPE) $\geq 18$ on Borg's category RPE scale (Green et al., 2003). Heart rate was measured using a finger pulse oximeter (Zyklusmed CMS50DL, Zyklusmed, Hamburg, Germany).

\section{Blood samples}

Participants were requested to avoid using of supplements, and any strenuous exercise at least 48 hours prior to each blood sample. Venous blood samples were taken from the antecubital vein in the morning between 7:30 a.m. and 09:00 a.m. after 12-h fasting and then collected into Serum Tubes. Samples were left to coagulate at room temperature and then centrifuged for $10 \mathrm{~min}$ at $3000 \mathrm{rpm}$, after which serum was removed and frozen at $-28{ }^{\circ} \mathrm{C}$ and remained so until analyzed. The irisin and myostatin concentration were analyzed with a Hitachi 902 auto analyzer (Hitachi, Tokyo, Japan), using irisin and myostatin hormone-specific immune-assay kits (ZellBio GmbH, Ulm, Germany) and optical density was measured at $450 \mathrm{~nm}$. The intraassay and inter-assay coefficient of variation was $<10 \%$ and $<12 \%$, respectively. Sensitivities for irisin and myostatin were $0.02 \mu \mathrm{g} / \mathrm{ml}$ and $2 \mathrm{ng} / \mathrm{l}$, respectively.

\section{Statistical analyses}

Data were presented as mean $\pm S D$. Normality of the data was evaluated and confirmed by the Shapiro-Wilk tests. Weight, waist circumference, hip circumference, $\mathrm{VO}_{2 \max }$, and leg press strength showed normal distribution; however, waist to hip ratio (WHR), body mass index (BMI), serum irisin, and myostatin did not have normal distribution. To compare four groups' performances, a one-way analysis of covariance (ANCOVA) was used, controlling for covariates of baseline value and using one-way ANOVA if pre-test have normal distribution. LSD post hoc tests were employed when the difference was significant $(p<.05)$ based on to the results of the ANCOVA or ANOVA analysis. Moreover, within-groups changes were done using paired sample $t$-tests for normal variables. For non-normal distribution variables, the comparisons of between groups at the baseline and post, and within groups were made using the Kruskal Wallis and Wilcoxon test, respectively. Spearman's rank correlation test was used to test the relationship between serum irisin and myostatin. The SPSS software (Version 22.0 for Windows; IBM, Armonk, NY, USA) was used for statistical analyses. The significance level was set to $\alpha=.05$. 


\section{Results}

Participant baseline and between-groups interaction of body composition are shown in Table 1. Significant differences were observed in waist circumference $(p=.003)$ and WHR $(p=.04)$ at the baseline between the study groups. There were no significant differences between groups at the baseline for body weight $(p=.19)$, BMI $(p=.14)$, and hip circumference $(p=.13)$ and over the 8-week period with controlling for covariates of baseline for weight ( $p=.67)$; BMI $(p=.49)$; waist circumference $(p=.08)$ and hip circumference $(p=.89)$. In addition, it was found that WHR $(p=.29)$ and BMI $(p=.14)$ were not significantly differ between groups, based on the analysis of the post-test results. Inter-group changes indicated that weight and BMI was significantly improved in CET ( $p=.03, p=.01$, respectively). However, no changes were found in other groups for weight or BMI. In addition, There were no significant differences in waist circumference, hip circumference and WHR for RET, AET, CET and control group.

Inter-group assessments indicated that significant improvements in $\mathrm{VO}_{2 \max }$ for the AET and CET groups ( $p=.002$ and $p=.015$, respectively). Yet, there was no significant difference in the $\mathrm{VO}_{2 \max }$ level of the RET and control groups $(p>.05)$. There were significant

Table 1

Body composition and physical characteristics

\begin{tabular}{|c|c|c|c|c|c|c|}
\hline & \multirow{2}{*}{$\begin{array}{l}\text { Resistance group } \\
\qquad(n=10)\end{array}$} & \multirow{2}{*}{$\begin{array}{l}\text { Aerobic group } \\
\quad(n=9)\end{array}$} & \multirow{2}{*}{$\begin{array}{l}\text { Concurrent group } \\
\qquad(n=10)\end{array}$} & \multirow{2}{*}{$\begin{array}{l}\text { Control group } \\
\quad(n=6)\end{array}$} & \multicolumn{2}{|c|}{ Main effect $(N=35)$} \\
\hline & & & & & Baseline & 8 weeks \\
\hline \multicolumn{7}{|l|}{ Age (years) } \\
\hline Baseline & $24.60 \pm 2.45$ & $24.66 \pm 2.29$ & $26.60 \pm 4.00$ & $25.50 \pm 4.80$ & - & - \\
\hline 8 weeks & - & - & - & - & & \\
\hline \multicolumn{7}{|l|}{ Height $(\mathrm{cm})$} \\
\hline Baseline & $160.56 \pm 5.96$ & $161.02 \pm 6.76$ & $160.42 \pm 5.67$ & $161.81 \pm 2.45$ & - & - \\
\hline 8 weeks & - & - & - & - & & \\
\hline \multicolumn{7}{|l|}{ Weight (kg) } \\
\hline Baseline & $60.51 \pm 8.12$ & $69.35 \pm 9.46$ & $63.95 \pm 11.00$ & $61.03 \pm 7.67$ & $* * \$$ & $\mathrm{I}$ \\
\hline 8 weeks & $60.54 \pm 8.00$ & $68.95 \pm 8.30$ & $63.44 \pm 10.99 *$ & $61.20 \pm 7.67$ & & \\
\hline \multicolumn{7}{|c|}{ Body mass index $\left(\mathrm{kg} / \mathrm{m}^{2}\right)$} \\
\hline Baseline & $23.45 \pm 2.83$ & $26.70 \pm 2.70$ & $24.87 \pm 4.21$ & $23.28 \pm 2.62$ & $* * €$ & $\$$ \\
\hline 8 weeks & $23.42 \pm 2.70$ & $26.49 \pm 2.39$ & $24.58 \pm 4.08 ¥$ & $23.38 \pm 2.62$ & & \\
\hline \multicolumn{7}{|c|}{ Waist circumference (cm) } \\
\hline Baseline & $71.90 \pm 4.04$ & $81.44 \pm 5.97$ & $81.00 \pm 8.40$ & $74.50 \pm 3.01$ & $* * \$$ & I \\
\hline 8 weeks & $71.80 \pm 3.51$ & $81.66 \pm 5.38$ & $79.50 \pm 7.83$ & $74.50 \pm 2.79$ & & \\
\hline \multicolumn{7}{|c|}{ Hip circumference $(\mathrm{cm})$} \\
\hline Baseline & $96.00 \pm 5.84$ & $103.38 \pm 4.51$ & $101.40 \pm 7.56$ & $99.83 \pm 9.78$ & $* * \$$ & $\mathrm{I}$ \\
\hline 8 weeks & $96.07 \pm 5.63$ & $103.41 \pm 4.42$ & $101.40 \pm 6.76$ & $100.08 \pm 9.74$ & & \\
\hline \multicolumn{7}{|c|}{ Waist-hip ratio (ratio) } \\
\hline Baseline & $0.74 \pm 0.03$ & $0.78 \pm 0.04$ & $0.79 \pm 0.03$ & $0.75 \pm 0.05$ & $* * €$ & $€$ \\
\hline 8 weeks & $0.74 \pm 0.03$ & $0.78 \pm 0.03$ & $0.78 \pm 0.04$ & $.074 \pm 0.05$ & & \\
\hline \multicolumn{7}{|c|}{$\mathrm{VO}_{2 \max }(\mathrm{ml} / \mathrm{kg} / \mathrm{min})$} \\
\hline Baseline & $34.46 \pm 1.64$ & $33.16 \pm 2.06$ & $31.92 \pm 1.30$ & $37.16 \pm 1.95$ & $* * \$$ & $* * \mathrm{I}$ \\
\hline 8 weeks & $34.77 \pm 1.44 \ddagger$ & $37.60 \pm 2.50 * \dagger$ & $33.04 \pm 1.26 * \dagger \div$ & $35.97 \pm 2.21$ & & \\
\hline \multicolumn{7}{|c|}{ Leg press 1RM (kg) } \\
\hline Baseline & $48.42 \pm 15.84$ & $47.34 \pm 3.86$ & $46.72 \pm 5.00$ & $48.01 \pm 11.45$ & $* * \$$ & $* * \mathrm{I}$ \\
\hline 8 weeks & $61.07 \pm 14.80 * \dagger+\S$ & $51.39 \pm 4.17^{*}$ & $52.55 \pm 5.85^{*}$ & $48.82 \pm 11.85$ & & \\
\hline
\end{tabular}

$¥$ Wilcoxon test showed significantly different value from the baseline value in the same group $(p<.05)$. * $t$-test showed significantly different value from the baseline value in the same group $(p<.05)$. † Significantly different from the control group at the same time point $(p<.05)$. $\neq$ Significantly different from the aerobic exercise group at the same time point $(p<.05)$. § Significantly between resistance and concurrent exercise group at the same time point $(p<.05)$. \$ 1-way ANOVA interaction between groups. I 1-way ANCOVA interaction for group adjusted for baseline. $€$ Kruskal-Wallis interaction between groups. ${ }^{* *}$ Significantly different between groups at the same time point $(p<.05)$. 
differences between groups in changes in $\mathrm{VO}_{2 \max }$ $(p<.001)$, where the AET group differed from the RET and control groups $(p=.001)$. These suggested that $\mathrm{VO}_{2 \max }$ significantly improved in both AET and CET groups, but it was grater in the AET group.

Inter-group evaluation showed significant improvement for leg press strength in the RET, AET, and CET groups $(p<.001, p=.003$, and $p<.001$, respectively). There was no significant difference in the control group's leg press strength $(p>.05)$. Furthermore, the results showed a significant interaction effect between the study groups based on the results of the leg press strength in post-test $(p<.001)$. RET group showed more improvement compared to all the other groups (RET vs. AET: $p=.041$, RET vs. CET: $p=.006$, and RET vs. control: $p<.001$; Table 1).

There were no significant differences between groups in terms of changes caused by serum irisin and myostatin at the baseline and after the 8-week period. However, the CET and RET groups had inter- group
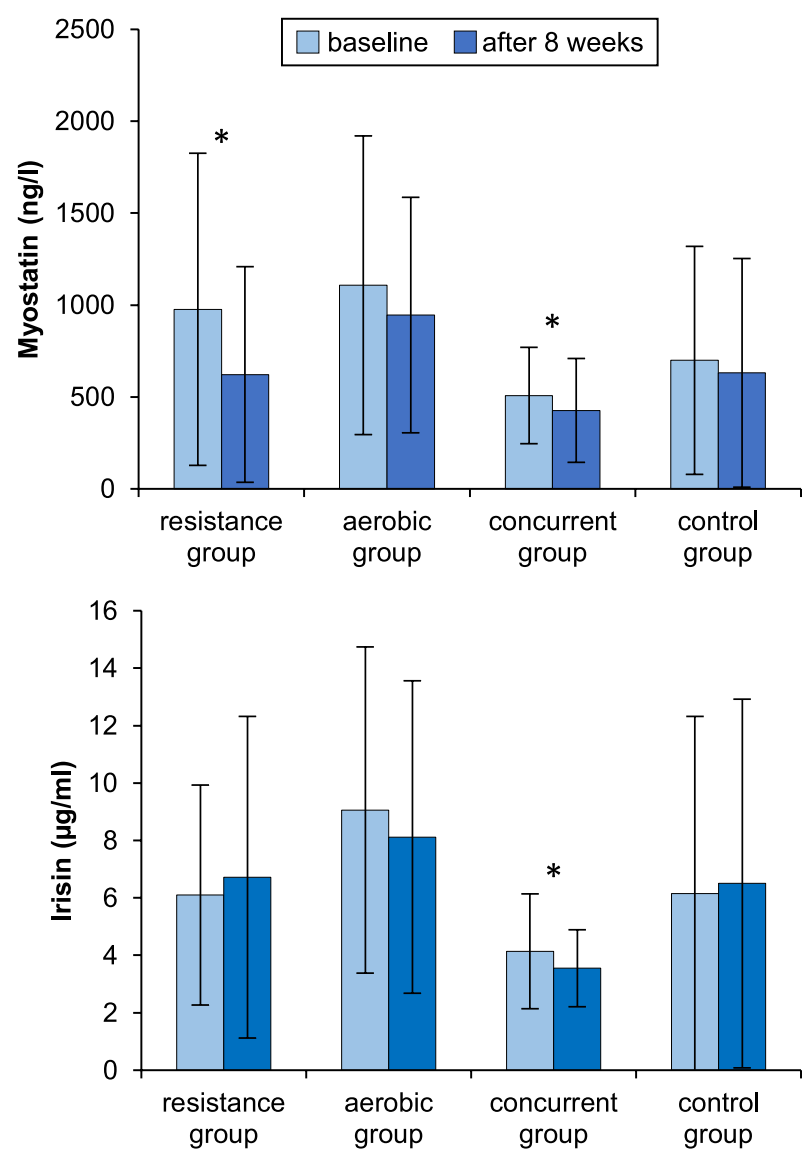

Figure 1. Changes of circulating myostatin and irisin in resistance $(n=10)$, aerobic $(n=9)$, concurrent $(n=10)$, and control $(n=6)$ groups from the baseline to after 8 weeks of training. Data are presented as mean $\pm S D$. * denotes statistically significant difference from the baseline in the same group. changes in the expected direction for myostatin ( $p=.005$ and $p=.008$, respectively). There were no statistically significant changes in the AET and control groups $(p>.05)$. Serum irisin was decreased from the first to 8 -week in CET group ( $p=.028$ ). There were no significant changes in the other groups ( $p>.05$; see Figure 1).

At the baseline, irisin positively correlated with myostatin $(r=.87, p<.001)$. In addition, irisin was positively correlated with myostatin after the 8-week AET $(r=.94, p<.001)$, RET $(r=.78, p=.004)$, and CET $(r=.90, p<.001)$. Irisin and myostatin correlation is illustrated in Figure 2.

\section{Discussion}

Muscle tissue constitutes $40 \%$ of the total body mass in non-obese mammals and accounts for $\sim 30 \%$ of the resting metabolic rate in adult humans (Zurlo, Larson, Bogardus, \& Ravussin, 1990), representing the largest
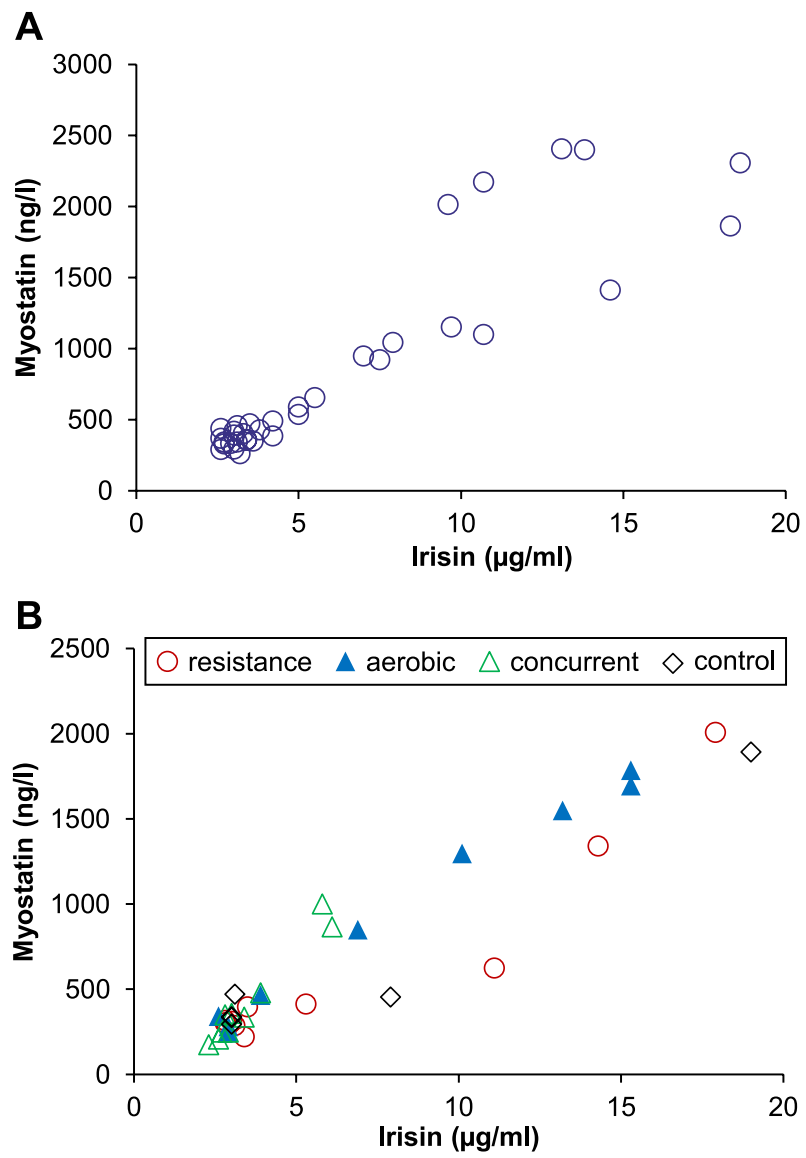

Figure 2. Correlation between circulating irisin and myostatin at the baseline (A) and after eight weeks of aerobic, resistance, or concurrent exercise training (B) in healthy untrained women. 
organ of the whole organism. Training induced muscular adaptations are reflected by changes in contractile protein and function, mitochondrial function, metabolic regulation, intracellular signaling, and transcriptional responses (Widrick, Stelzer, Shoepe, \& Garner, 2002) and these changes can be mediated by myokines.

The aim of this study was to evaluate the effect of an 8 week AET, RET or CET training period on irisin and myostatin circulation in healthy untrained women. Results showed that RET and AET have no effect on serum irisin in previously untrained young women. Serum irisin level was significantly decreased in the concurrent group. The findings of several studies are in favor of the present study findings. These studies found that RET (Ellefsen et al., 2014; Hecksteden et al., 2013; Moienneia \& Hosseini, 2016) and AET (Hecksteden et al., 2013; Pekkala et al., 2013) did not have an effect on circulating irisin. Notwithstanding, there is limited information about the effects of CET on circulating irisin. Pekkala et al. (2013) found CET did not have significant effect on irisin. Recent evidence suggested that circulating irisin levels were significantly correlated with BMI (Huh et al., 2012). In line with these, the researchers found a significant reduction of BMI in the CET group, which showed a decrease in irisin. Moreover, for the other two groups, no changes occurred in their BMI level. In this regard, Ercan et al. (2017) and Ghanbari-Niaki et al. (2018) showed an increased irisin level, which resulted from weight loss after 10 weeks of freewheel running exercise in female mice and eight weeks of resistance training in postmenopausal women, respectively. On the other hand, Boström et al. (2012) reported a twofold increase in circulation irisin after 10 weeks of AET in healthy overweight adults. However, because of the low subject number $(N=8)$ and lack of a control group, this result warrants confirmation.

Furthermore, studies in aging mice and humans revealed that circulating irisin significantly increased following RET (Kim et al., 2015; Zhao et al., 2017). It seems that differences in the participants' age range can be considered as a reason for the differences in results.

The role of irisin in metabolism reflects itself in an increase in energy expenditure and glucose homeostasis (Boström et al., 2012). Therefore, it would be logical that irisin and FNDC5 increase in response to AET is generally characterized by increased oxidative capacity and mitochondrial functions. Also, it is possible that the anabolic effects (muscle growth) of RET are associated with increased FNDC5 expression since in a study authors showed that irisin was mostly associated with muscle mass (Huh et al., 2012). The results of this research demonstrated the improvement of $\mathrm{VO}_{2 \max }$ (in the AET and CET groups) and muscle strength (due to greater muscle mass in RET and CET). Irisin levels not increasing during the study can be explained with reference to Raschke et al. (2013). These researchers showed that a difference between the human and murine initiation codon led to the majority of translated human FNDC5 proteins to lose their signal peptides, and this caused the production of about $1 \%$ full length protein.

To best of our knowledge, there is no evidence about the effect of CET on circulating myostatin. Furthermore, the present study is the first study on circulating myostatin in humans that compared three types of exercise training. Based on the present study results, RET and CET showed a significant effect in decreasing circulating myostatin, while AET did not produce an effect on circulating myostatin. In line with these findings, studies have reported that circulating myostatin decreased after RET (Saremi et al., 2010; Walker et al., 2004). Although, Hofmann et al. (2016) and Binns et al. (2017) showed that RET in aged human have no significant effect on serum myostatin. The contradictory results found in these studies are possibly due to the participants' age and exercise protocol. In the present study we used young women and the RET was performed over eight weeks and for three times per week at $65-75 \%$ 1RM. However, the aforementioned studies were focused on older men and women, with a 6-month or 5-month exercise training period, and running twice a week at $20-30 \%$ or $50-70 \%$ 1RM program was used, respectively. In a study on middle aged men, authors demonstrated that moderate AET reduced plasma myostatin levels (Hittel et al., 2010). There is little literature on the effects of exercise training on circulating myostatin, despite that fact, there are a great deal of studies that have examined the relationship between myostatin expression and exercise training. Similar to RET (Roth et al., 2003), AET are usually characterized by a decrease in myostatin expression (Besse-Patin et al., 2014; Ziaaldini et al., 2015). The controversial results from the present study may be due to a different protocol of exercise training. According to that, myostatin tend to decrease in the AET group, and it seems that exercise duration or intensity were not enough for the study population. Since, in the present study, RET reduced circulating myostatin and AET result in tendency to decrease, it is not surprising that CET also reduced circulating myostatin levels. myostatin is a member of the transforming growth factor-beta protein (TGF- $\beta$ ) family which is secreted from muscle cells and acts in an auto/paracrine manner by inhibiting Satellite cells proliferation and differentiation, and by blocking muscle fiber protein accretion (Allen, Hittel, \& McPherron, 2011). Despite the negative effect of myostatin on 
muscle growth and development (McPherron et al., 1997), myostatin has effect on homeostasis. myostatin mediates some metabolic condition, such shift from lipid oxidation towards glycolysis, reduced mitochondrial capacity and insulin resistance (Bergouignan, Rudwill, Simon, \& Blanc, 2011). Myostatin seems to have a biological role also in the crosstalk between skeletal muscle and adipose tissue and seem to be involved in insulin sensitivity (Argilés, Orpí, Busquets, \& LópezSoriano, 2012), although it has been suggested that myostatin does not directly act on adipocytes function and metabolism, but that increased muscle mass leads to a higher energy expenditure, greater lipid uptake, and more active metabolism, finally decreasing the mass of adipose tissue (Allen et al., 2011).

In addition, the present study found that irisin was directly associated with myostatin in untrained women (based on the pre-test results). The results of the present study also showed a direct correlation in trained women (after the 8-weeks exercise training). A recent study in human adipocytes demonstrates that irisin treatment increases IGF-1 and decreases myostatin mRNA levels, the two main factors for muscle growth (Huh et al., 2014). Another study has suggested that irisin and myostatin levels could inverse associated and activating energy metabolism and thermogenesis in diabetic patients (García-Fontana et al., 2016). It seems that the contradictory results found in our study are associated with the characteristics of the participants studied. Huh et al. (2014) assessed the correlation in healthy obese participants, and the irisin correlation was conducted with myostatin mRNA levels. In addition, the second study was conducted in diabetic patients. The participants of the present study were healthy normal or overweight. Also the small number of participants can account for the low dependability of the findings of the present study.

Among possible limitations of the present study we can consider the possible influence of macronutrient intake. It was suggested that circulating irisin levels significantly correlate with carbohydrate intake (Lopez-Legarrea et al., 2014) as well as energy restriction (Iglesia et al., 2014). A further limitation should be considered that the participants' diet was not supervised and the small sample size of the control group, which needs evaluation in future studies.

\section{Conclusion}

In conclusion, a general induction of irisin by regular exercise training seems unlikely. According to the results, RET and AET did not produce a noticeable effect on irisin. In contrast, CET results in statistically decreased irisin level. It seems that CET is more effective than AET or RET on irisin concentration. Serum myostatin was reduced after eight weeks of RET or CET implementation. In addition, circulating myostatin did not alter in response to AET. Finally, a strong positive correlation was found between circulating irisin and myostatin in untrained participants or after 8 weeks of exercise training.

\section{Acknowledgments}

This work was supported by University of Islamic Azad University Rasht Branch. The authors would like to thank the subjects who participated in this study.

\section{Conflict of interest}

There were no conflicts of interest.

\section{References}

Allen, D. L., Hittel, D. S., \& McPherron, A. C. (2011). Expression and function of myostatin in obesity, diabetes, and exercise adaptation. Medicine \& Science in Sports \& Exercise, 43, 1828-1835.

Argilés, J. M., Orpí, M., Busquets, S., \& López-Soriano, F. J. (2012). Myostatin: More than just a regulator of muscle mass. Drug Discovery Today, 17, 702-709.

Bastos, C. L. B., Miranda, H., de Souza Vale, R. G., de Nazaré Portal, M., Gomes, M. T., da Silva Novaes, J., \& Winchester, J. B. (2013). Chronic effect of static stretching on strength performance and basal serum IGF-1 levels. Journal of Strength and Conditioning Research, 27, 2465-2472.

Bergouignan, A., Rudwill, F., Simon, C., \& Blanc, S. (2011). Physical inactivity as the culprit of metabolic inflexibility: Evidence from bed-rest studies. Journal of Applied Physiology, 111, 1201-1210.

Besse-Patin, A., Montastier, E., Vinel, C., Castan-Laurell, I., Louche, K., Dray, C., ... Thalamas, C. (2014). Effect of endurance training on skeletal muscle myokine expression in obese men: Identification of apelin as a novel myokine. International Journal of Obesity, 38, 707-713.

Binns, A., Gray, M., Henson, A. C., \& Fort, I. L. (2017). Changes in lean mass and serum myostatin with habitual protein intake and high-velocity resistance training. Journal of Nutrition, Health \& Aging, 21, 1111-1117.

Boström, P., Wu, J., Jedrychowski, M. P., Korde, A., Ye, L., Lo, J. C., ... Long, J. Z. (2012). A PGC1- $\alpha$-dependent myokine that drives brown-fat-like development of white fat and thermogenesis. Nature, 481, 463-468.

Bruce, R. A., Kusumi, F., \& Hosmer, D. (1973). Maximal oxygen intake and nomographic assessment of functional aerobic impairment in cardiovascular disease. American Heart Journal, 85, 546-562. 
Ellefsen, S., Vikmoen, O., Slettaløkken, G., Whist, J. E., Nygård, H., Hollan, I., ... Raastad, T. (2014). Irisin and FNDC5: Effects of 12-week strength training, and relations to muscle phenotype and body mass composition in untrained women. European Journal of Applied Physiology, 114, 1875-1888.

Ercan, B., Umit, Z., Ebru Gurel, G., Bahar Yüksel, O., Faruk, C., Nazli, O., ... John, Y. (2017). Effects of irisin and exercise on metabolic parameters and reproductive hormone levels in high-fat diet-induced obese female mice. Reproductive Sciences, 25, 281-291.

Garber, C. E., Blissmer, B., Deschenes, M. R., Franklin, B. A., Lamonte, M. J., Lee, I.-M., ... Swain, D. P. (2011). American College of Sports Medicine position stand. Quantity and quality of exercise for developing and maintaining cardiorespiratory, musculoskeletal, and neuromotor fitness in apparently healthy adults: Guidance for prescribing exercise. Medicine \& Science in Sports \& Exercise, 43, 1334-1359.

García-Fontana, B., Reyes-García, R., Morales-Santana, S., Ávila-Rubio, V., Muñoz-Garach, A., Rozas-Moreno, P., \& Muñoz-Torres, M. (2016). Relationship between myostatin and irisin in type 2 diabetes mellitus: A compensatory mechanism to an unfavourable metabolic state? Endocrine, 52, 54-62.

Ghanbari-Niaki, A., Saeidi, A., Ahmadian, M., Gharahcholo, L., Naghavi, N., Fazelzadeh, M., ... Williams, A. (2018). The combination of exercise training and Zataria multiflora supplementation increase serum irisin levels in postmenopausal women. Integrative Medicine Research, 7, 44-52.

Green, J. M., Crews, T. R., Bosak, A. M., \& Peveler, W. W. (2003). A comparison of respiratory compensation thresholds of anaerobic competitors, aerobic competitors and untrained subjects. European Journal of Applied Physiology, 90, 608-613.

Hecksteden, A., Wegmann, M., Steffen, A., Kraushaar, J., Morsch, A., Ruppenthal, S., ... Meyer, T. (2013). Irisin and exercise training in humans - results from a randomized controlled training trial. BMC Medicine, 11, 235.

Hinkley, J. M., Konopka, A. R., Suer, M. K., \& Harber, M. P. (2017). Short-term intense exercise training reduces stress markers and alters the transcriptional response to exercise in skeletal muscle. American Journal of Physiology - Regulatory, Integrative and Comparative Physiology, 312, R426-R433.

Hittel, D. S., Axelson, M., Sarna, N., Shearer, J., Huffman, K. M., \& Kraus, W. E. (2010). Myostatin decreases with aerobic exercise and associates with insulin resistance. Medicine \& Science in Sports \& Exercise, 42, 2023-2029.

Hofmann, M., Schober-Halper, B., Oesen, S., Franzke, B., Tschan, H., Bachl, N., ... Wessner, B. (2016). Effects of elastic band resistance training and nutritional supplementation on muscle quality and circulating muscle growth and degradation factors of institutionalized elderly women: The Vienna Active Ageing Study (VAAS). European Journal of Applied Physiology, 116, 885-897.

Huh, J. Y., Dincer, F., Mesfum, E., \& Mantzoros, C. (2014). Irisin stimulates muscle growth-related genes and regulates adipocyte differentiation and metabolism in humans. International Journal of Obesity, 38, 1538-1544.
Huh, J. Y., Panagiotou, G., Mougios, V., Brinkoetter, M., Vamvini, M. T., Schneider, B. E., \& Mantzoros, C. S. (2012). FNDC5 and irisin in humans: I. Predictors of circulating concentrations in serum and plasma and II. mRNA expression and circulating concentrations in response to weight loss and exercise. Metabolism, 61, 1725-1738.

Iglesia, R., Lopez-Legarrea, P., Crujeiras, A. B., Pardo, M., Casanueva, F. F., Zulet, M. A., \& Martinez, J. A. (2014). Plasma irisin depletion under energy restriction is associated with improvements in lipid profile in metabolic syndrome patients. Clinical Endocrinology, 81, 306-311.

Karsenty, G., \& Olson, E. N. (2016). Bone and muscle endocrine functions: Unexpected paradigms of inter-organ communication. Cell, 164, 1248-1256.

Kim, H.-J., So, B., Choi, M., Kang, D., \& Song, W. (2015). Resistance exercise training increases the expression of irisin concomitant with improvement of muscle function in aging mice and humans. Experimental Gerontology, 70, 11-17.

Kurdiova, T., Balaz, M., Vician, M., Maderova, D., Vlcek, M., Valkovic, L., ... Belan, V. (2014). Effects of obesity, diabetes and exercise on Fndc5 gene expression and irisin release in human skeletal muscle and adipose tissue: In vivo and in vitro studies. Journal of Physiology, 592, 1091-1107.

Lopez-Legarrea, P., De La Iglesia, R., Crujeiras, A. B., Pardo, M., Casanueva, F. F., Zulet, M., \& Martinez, J. A. (2014). Higher baseline irisin concentrations are associated with greater reductions in glycemia and insulinemia after weight loss in obese subjects. Nutrition \& Diabetes, 4, e110.

McPherron, A. C., Lawler, A. M., \& Lee, S.-J. (1997). Regulation of skeletal muscle mass in mice by a new TGF-beta superfamily member. Nature, 387, 83-90.

Moienneia, N., \& Hosseini, S. R. A. (2016). Acute and chronic responses of metabolic myokine to different intensities of exercise in sedentary young women. Obesity Medicine, 1, 15-20.

Mouisel, E., Relizani, K., Mille-Hamard, L., Denis, R., Hourde, C., Agbulut, O., ... Vignaud, A. (2014). Myostatin is a key mediator between energy metabolism and endurance capacity of skeletal muscle. American Journal of Physiology - Regulatory, Integrative and Comparative Physiology, 307, R444-R454.

Nascimento, M. A., Cyrino, E. S., Nakamura, F. Y., Romanzini, M., Pianca, H. J. C., \& Queiróga, M. R. (2007). Validation of the Brzycki equation for the estimation of 1-RM in the bench press. Revista Brasileira de Medicina do Esporte, 13, 47-50.

Pekkala, S., Wiklund, P. K., Hulmi, J. J., Ahtiainen, J. P., Horttanainen, M., Pöllänen, E., ... Nyman, K. (2013). Are skeletal muscle FNDC5 gene expression and irisin release regulated by exercise and related to health? Journal of Physiology, 591, 5393-5400.

Raschke, S., Elsen, M., Gassenhuber, H., Sommerfeld, M., Schwahn, U., Brockmann, B., ... Raastad, T. (2013). Evidence against a beneficial effect of irisin in humans. PloS ONE, 8, e73680.

Ratamess, N., Alvar, B., Evetoch, T., Housh, T., Kibler, W., \& Kraemer, W. (2009). American College of Sports Medicine position stand. Progression models in resistance 
training for healthy adults. Medicine \& Science in Sports \& Exercise, 41, 687-708.

Rodgers, B. D., \& Garikipati, D. K. (2008). Clinical, agricultural, and evolutionary biology of myostatin: A comparative review. Endocrine Reviews, 29, 513-534.

Roth, S. M., Martel, G. F., Ferrell, R. E., Metter, E. J., Hurley, B. F., \& Rogers, M. A. (2003). Myostatin gene expression is reduced in humans with heavy-resistance strength training: A brief communication. Experimental Biology and Medicine, 228, 706-709.

Sanchis-Gomar, F., \& Perez-Quilis, C. (2014). The p38PGC-1 $\alpha$-irisin-betatrophin axis: Exploring new pathways in insulin resistance. Adipocyte, 3, 67-68.

Saremi, A., Gharakhanloo, R., Sharghi, S., Gharaati, M., Larijani, B., \& Omidfar, K. (2010). Effects of oral creatine and resistance training on serum myostatin and GASP-1. Molecular and Cellular Endocrinology, 317, 25-30.

Spiegelman, B. M. (2013). Banting Lecture 2012: Regulation of adipogenesis: Toward new therapeutics for metabolic disease. Diabetes, 62, 1774-1782.
Walker, K. S., Kambadur, R., Sharma, M., \& Smith, H. K. (2004). Resistance training alters plasma myostatin but not IGF-1 in healthy men. Medicine \& Science in Sports \& Exercise, 36, 787-793.

Widrick, J. J., Stelzer, J. E., Shoepe, T. C., \& Garner, D. P. (2002). Functional properties of human muscle fibers after short-term resistance exercise training. American Journal of Physiology - Regulatory, Integrative and Comparative Physiology, 283, R408-R416.

Zhao, J., Su, Z., Qu, C., \& Dong, Y. (2017). Effects of 12 weeks resistance training on serum irisin in older male adults. Frontiers in Physiology, 8, 171.

Ziaaldini, M. M., Koltai, E., Csende, Z., Goto, S., Boldogh, I., Taylor, A. W., \& Radak, Z. (2015). Exercise training increases anabolic and attenuates catabolic and apoptotic processes in aged skeletal muscle of male rats. Experimental Gerontology, 67, 9-14.

Zurlo, F., Larson, K., Bogardus, C., \& Ravussin, E. (1990). Skeletal muscle metabolism is a major determinant of resting energy expenditure. Journal of Clinical Investigation, 86, 1423-1427. 